\title{
Articles
}

\section{Rosiglitazone short-term monotherapy lowers fasting and post-prandial glucose in patients with Type II diabetes}

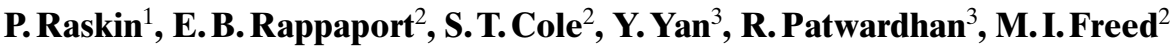 \\ ${ }^{1}$ University of Texas Southwestern Medical Center, Dallas, Texas, USA \\ ${ }^{2}$ Clinical Research, Development and Medical Affairs North America, SmithKline Beecham Pharmaceuticals, Collegeville, \\ Pennsylvania, USA \\ ${ }^{3}$ Biometrics, SmithKline Beecham Pharmaceuticals, Collegeville, Pennsylvania, USA
}

\section{Abstract}

Aims/hypothesis. The short-term efficacy, safety and tolerability of rosiglitazone were compared with placebo in patients with Type II (non-insulin-dependent) diabetes mellitus in a dose-ranging study.

Methods. After a 2-week placebo run-in phase, 303 patients were randomly assigned to 8 weeks of treatment with twice-daily placebo or 2, 4 or $6 \mathrm{mg}$ of rosiglitazone.

Results. All rosiglitazone doses significantly reduced fasting plasma glucose compared with baseline. All rosiglitazone treatment groups showed significantly reduced peak postprandial glucose concentrations compared with baseline $(p<0.001)$ and with placebo $(p<0.0001)$ and reduced postprandial glucose excursion, without an increase in the area under the postprandial insulin concentration-time curve. Rosiglitazone at 4 and $6 \mathrm{mg}$ twice daily prevented the increase in $\mathrm{HbA}_{1 \mathrm{c}}$ observed in the placebo group. $\mathrm{C}$ peptide and serum insulin concentrations were significantly reduced from baseline in all rosiglitazone treatment groups. In all rosiglitazone treatment groups, nonesterified fatty acids decreased significantly $(p<$ 0.0001 ) and triglycerides did not change. Although total LDL and HDL cholesterol increased significantly in the rosiglitazone treatment groups, total cholesterol/HDL ratios did not change significantly. The proportion of patients with one or more adverse event was similar in all four treatment groups. No patient showed evidence of hepatotoxicity.

Conclusion/interpretation. Rosiglitazone given twice daily significantly reduced fasting and postprandial glucose concentrations, $\mathrm{C}$ peptide, insulin and nonesterified fatty acids in Type II diabetic patients. The glucose-lowering effect of the 4-mg twice-daily dose of rosiglitazone was similar to that of 6-mg twice daily, suggesting that $4 \mathrm{mg}$ twice daily should be the maximum clinical dose. [Diabetologia (2000) 43: 278-284]

Keywords Rosiglitazone, thiazolidinedione, insulin resistance, Type II diabetes, non-esterified fatty acids, efficacy.
Because insulin resistance plays a prominent part in the development of Type II diabetes $[1,2]$, therapeutic interventions to improve insulin action are likely

Received: 15 July 1999 and in revised form: 30 September 1999

Corresponding author: P. Raskin, MD, University of Texas Southwestern Medical Center, 5323 Harry Hines Blvd, Rm G5.238, Dallas, TX 75390-8858, USA

Abbreviations: ALT, Alanine aminotransferase; AST, aspartate aminotransferase; FPG, fasting plasma glucose; PPAR $\gamma$, peroxisome proliferator-activated receptor-gamma; PPG, post-prandial glucose. to be of considerable benefit in the management of the condition. The thiazolidinediones (or glitazones) are a new class of orally active drugs that reduce insulin resistance [3] and hence increase glucose uptake in skeletal muscle and adipose tissue, as well as decrease hepatic glucose production. These effects are thought to be mediated through interactions of these drugs with the gamma subtype of the peroxisome proliferator-activated receptor gamma (PPAR $\gamma)[4,5]$.

The thiazolidinediones currently marketed, or in late-phase clinical trials, include troglitazone, rosiglitazone and pioglitazone. Although these compounds share a common thiazolidine-2-4-dione structure, 
Table 1. Baseline demographic and metabolic characteristics

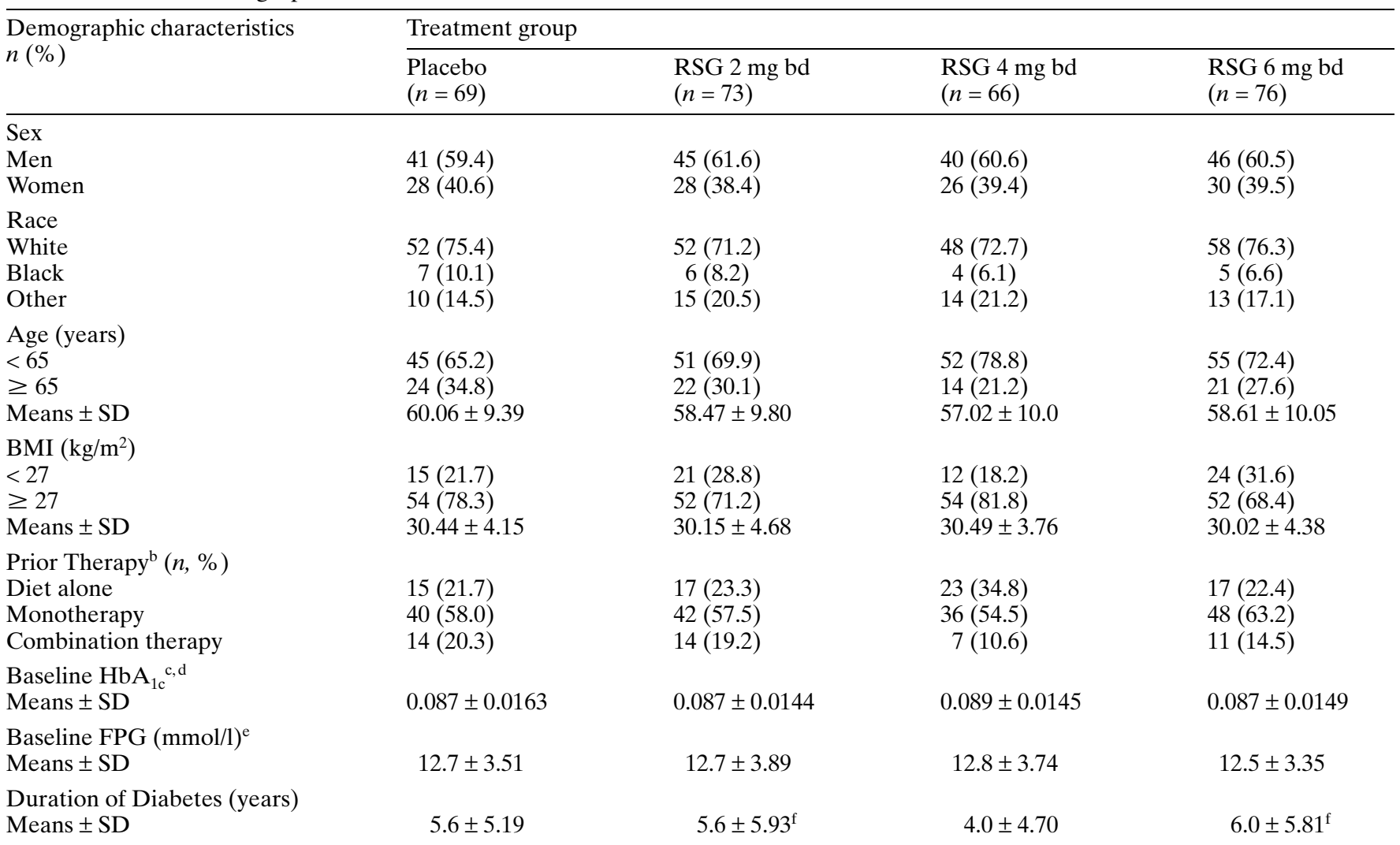

a Three patients younger than 40 years were allowed to enter the study after confirmation with the sponsor: two in the 2-mg bd group and one in the 4-mg bd group.

${ }^{b}$ In the 3 months before the screening visit.

${ }^{c}$ Reference range: $<0.065$.

differences in the side chains result in differences in bioavailability, metabolism and antihyperglycaemic action [5]. Rosiglitazone is the most potent agent in the class, being 100 times more potent than troglitazone in terms of its activation of PPAR $\gamma$ [4]. A previous dose-ranging study has adequately described the safety and efficacy of rosiglitazone at the lower end of the dose-response curve [6]. This study was designed to compare the effects of rosiglitazone given at a dose range of 2, 4 and $6 \mathrm{mg}$ twice daily versus placebo in individuals with Type II diabetes and to investigate the effects of rosiglitazone on postprandial glucose excursions.

\section{Subjects and methods}

Subjects. Patients were eligible for the study if they were 40 to 80 years of age and had Type II (non-insulin-dependent) diabetes mellitus (defined by the National Diabetes Data Group [7]), a fasting plasma glucose (FPG) concentration $7.8 \mathrm{mmol} / \mathrm{l}$ or more and $16.7 \mathrm{mmol} / \mathrm{l}$ or more and evidence of residual insulin secretory capacity as determined by a fasting C-peptide concentration $0.27 \mathrm{nmol} / \mathrm{l}$ or more (Table 1 ). Patients with clin- ically important renal (serum creatinine $>160 \mu \mathrm{mol} / \mathrm{l}$ ) or hepatic disease (ALT, AST, total bilirubin or alkaline phosphatase $>2.5$ times upper limit of the normal range), symptomatic angina pectoris or cardiac insufficiency (New York Heart Association functional class III or IV) or haematologic abnormalities were excluded from the study. Patients who required insulin therapy were not eligible for the study.

All patients were withdrawn from previous antidiabetic medication for 2 weeks before entrance into the study. During the 3 months before the study, $166(58.5 \%)$ of the patients had been treated with a single antihyperglycaemic agent. Approximately $25 \%$ were managed with diet alone and the remainder of the patients received therapy with more than one drug.

Study protocol. This multicentre, randomised, double-masked, placebo-controlled trial was conducted at 35 sites throughout the United States. All patients received placebo twice daily by mouth for 2 weeks during the single-masked run-in period. Patients were then randomly assigned to 8 weeks of doublemasked treatment with placebo or with rosiglitazone at doses of $2 \mathrm{mg}, 4 \mathrm{mg}$ or $6 \mathrm{mg}$ given twice daily. The study was conducted in accordance with the Declaration of Helsinki, Title 21 of the US Code of Federal Regulations and Good Clinical Practice guidelines. All study subjects gave written, informed consent before study enrolment. 

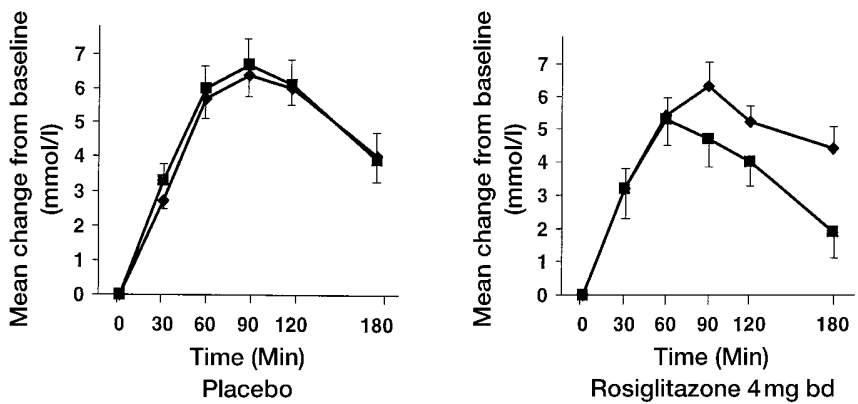

Fig.1. Rosiglitazone (RSG) significantly reduced fasting plasma glucose concentration after 8 weeks of treatment. All values are significantly different from baseline and all values from the rosiglitazone-treated groups are significantly different from placebo $(p<0.0001)$. Error bars are standard deviation. $\mathrm{bd}=$ twice daily

Postprandial analysis. To examine the effects of rosiglitazone on postprandial glucose, insulin and triglyceride concentrations, postprandial studies were carried out in approximately 4 patients at each site that chose to participate in this phase of the study. Each centre was considered a block in the randomization procedure so that patients who chose to undergo postprandial analysis would be distributed approximately equally to the four treatment groups. The assessments were made at baseline and week 8 , before and after a standard $500 \mathrm{kcal}$ breakfast. Study medication was taken in the clinic with the meal after all specimens for fasting analysis were obtained. Blood samples were obtained before breakfast and 30, 60, 90, 120 and $180 \mathrm{~min}$ after the start of breakfast.

Sample handling and laboratory measurements. Blood samples of approximately $7 \mathrm{ml}$ were collected from each patient into tubes containing EDTA. Plasma samples were obtained by centrifugation and stored at $-20^{\circ} \mathrm{C}$ for further analysis. Laboratory measurements for efficacy and safety were made by SmithKline Beecham Clinical Laboratories (Van Nuys, Calif., USA) on blood collected in the fasting state. Fasting plasma glucose, total cholesterol, HDL cholesterol and triglycerides were measured by an Olympus analyser (Olympus Clinical Instruments Division, Lake Success, N.Y., USA); $\mathrm{HbA}_{1 \mathrm{c}}$ by Variant, a high-performance liquid chromatography method (Bio-Rad, Hercules, Calif., USA); C peptide by radioimmunoassay (Diagnostic Products, Los Angeles, Calif., USA); insulin by radioimmunoassay (Pharmacia, Uppsala, Sweden); fructosamine by colorimetric analysis (RoTAG fructosamine assay, Roche Diagnostic Systems, Indianapolis, Ind., USA); and non-esterified fatty acids (NEFAs) by enzymatic/colorimetric analysis (Wako Diagnostic, Richmond, Va., USA) using a COBAS analyser (Roche Diagnostic Systems, Indianapolis, Ind., USA). LDL cholesterol concentrations were estimated from total and HDL cholesterol determinations using the Friedewald calculation [8]. Safety monitoring included physical examinations, vital signs, electrocardiograms, adverse experience query and clinical laboratory tests.

Statistical analysis. All statistical analysis was done using SAS for Windows (version 6.1). An analysis of covariance (ANCOVA) was used for the assessment of differences in continuous variables between the treatment groups. The results obtained using the full model including treatment-by-baseline and treatment-by-region interaction terms were compared with those obtained by a one-way analysis of variance (ANOVA) model; the conclusions were the same for both models.
Table 2. Fasting plasma glucose values at baseline and at 4 and 8 weeks $(\mathrm{mmol} / \mathrm{l})$

Time Placebo RSG $2 \mathrm{mg}$ bd RSG $4 \mathrm{mg}$ bd RSG $6 \mathrm{mg}$ bd

\begin{tabular}{llll}
$(n=69)$ & $(n=73)$ & $(n=66)$ & $(n=76)^{\mathrm{a}}$ \\
\hline Baseline $12.7 \pm 3.51$ & $12.7 \pm 3.89$ & $12.8 \pm 3.74$ & $12.5 \pm 3.35$
\end{tabular}

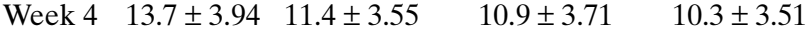

$\begin{array}{lllll}\text { Week } 8 & 13.8 \pm 3.93 & 10.7 \pm 3.59 & 10.4 \pm 3.56 & 10.0 \pm 3.49\end{array}$

${ }^{\mathrm{a}} n=74$ at 4 weeks and 75 at 8 weeks. bd = twice daily, $\mathrm{RSG}=$ rosiglitazone

Difference from baseline was assessed by paired Student's $t$ test. The Williams procedure was used to determine the minimum effective rosiglitazone dose compared with placebo. An intention-to-treat analysis with the last observation carried forward was used. Data are expressed as means $\pm \mathrm{SD} ; p$ values of 0.05 or less were considered to be statistically significant.

\section{Results}

Of the 529 patients screened, 303 were found to be eligible and were randomly assigned to double-masked treatment. Baseline demographic and metabolic characteristics were similar in the four treatment groups (Table 1).

Glycaemic control. Rosiglitazone significantly reduced mean fasting plasma glucose (FPG) concentration compared with both baseline and placebo (all $p<0.0001$ ). Improved glycaemic control was evident from the first visit following initiation of therapy. After 8 weeks of placebo treatment, the mean FPG increased significantly from a baseline value of $12.7 \pm$ $3.5 \mathrm{mmol} / \mathrm{l}$ to $13.8 \pm 3.9 \mathrm{mmol} / \mathrm{l}(p=0.0004)$. Rosiglitazone therapy significantly decreased FPG $(p<$ $0.0001)$ in the $2-\mathrm{mg}$ twice-daily $(12.7 \pm 3.9$ to $10.7 \pm$ $3.6 \mathrm{mmol} / \mathrm{l})$, $4-\mathrm{mg}$ twice-daily $(12.8 \pm 3.7$ to $10.4 \pm$ $3.6 \mathrm{mmol} / \mathrm{l})$ and $6-\mathrm{mg}$ twice-daily $(12.6 \pm 3.3$ to $10.0 \pm$ $3.5 \mathrm{mmol} / \mathrm{l}$ ) groups (Fig. 1, Table 2). The rate of decline in mean FPG was greatest during the first 4 weeks of treatment in all rosiglitazone treatment groups. Additional decreases in FPG were observed between weeks 4 and 8 .

Fructosamine. After 8 weeks of treatment, fructosamine concentrations had decreased significantly in the rosiglitazone 4 and 6-mg twice-daily treatment groups relative to baseline $(p<0.0001$ and $p=$ 0.0033 , respectively) and in all rosiglitazone treatment groups compared with placebo $(p<0.0001)$ (decreases of $3.3 \pm 61.8, \quad 27.2 \pm 46.36$ and $19.9 \pm$ $56.89 \mu \mathrm{mol} / 1$ in the rosiglitazone 2-, 4- and 6-mg twice-daily groups compared with an increase of $39.0 \pm 66.91 \mu \mathrm{mol} / 1$ in the placebo group).

Haemoglobin $A_{1 c}$. In the rosiglitazone 2-mg twicedaily treatment group and in the placebo group, 
Table 3. Changes in C peptide and insulin

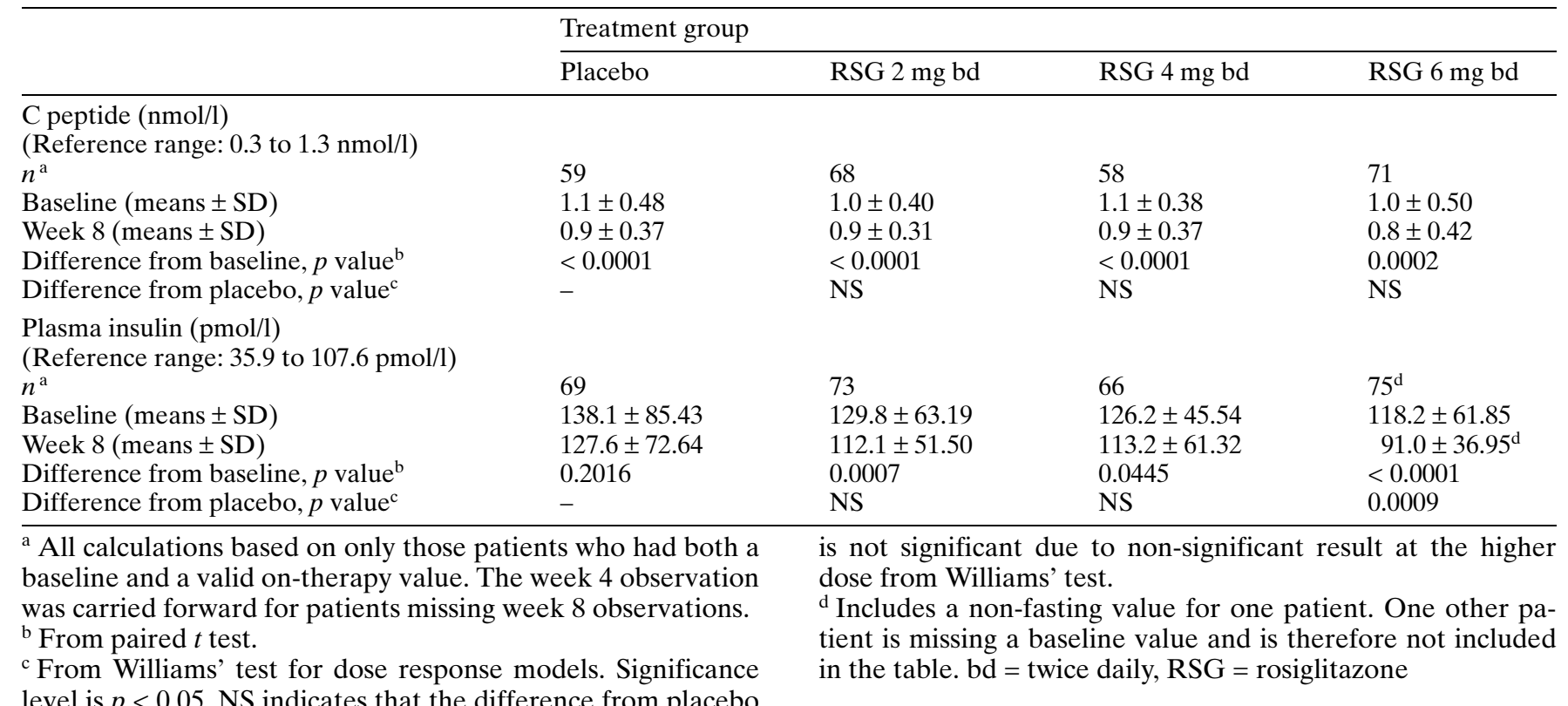

mean $\mathrm{HbA}_{1 \mathrm{c}}$ values increased significantly ( $p \leq 0.0025$ and $p<0.0001$, respectively) from baseline to week 8 (from $0.087 \pm 0.014$ to $0.091 \pm 0.018$ and from $0.087 \pm 0.016$ to $0.097 \pm 0.020$, respectively). In contrast, no increase occurred in patients treated with rosiglitazone at 4 and $6 \mathrm{mg}$ twice daily. For all doses of rosiglitazone, these changes differed significantly from the change in the placebo group ( $p<0.0001$ for $4 \mathrm{mg}$ and $6 \mathrm{mg}$ twice daily vs placebo; $p=0.0029$ for $2 \mathrm{mg}$ twice daily vs placebo).

Insulin and $C$ peptide. Insulin concentrations decreased significantly from baseline in rosiglitazonetreated patients $(p<0.05)$ but not in placebo-treated patients (Table 3). Rosiglitazone $6 \mathrm{mg}$ twice daily significantly reduced plasma insulin concentrations compared with placebo $(p<0.05)$. Mean $\mathrm{C}$ peptide concentrations decreased significantly from baseline in all of the rosiglitazone treatment groups (all $p \leq 0.0002$ ), although there was no significant difference from placebo.

Lipids. Rosiglitazone significantly reduced mean NEFA concentrations compared with baseline and placebo $(p<0.0001)$ (Table 4$)$. Mean increases in total cholesterol and LDL cholesterol for each rosiglitazone dose were significantly greater than those in the placebo group $(p<0.0001$ and $p<0.0004$, respectively). There were no mean changes in HDL compared with placebo and there were no clinically meaningful changes in mean total cholesterol:HDL or mean LDL:HDL ratios. Rosiglitazone did not affect mean triglyceride concentrations.
Postprandial analysis. The area under time-concentration curve (AUC) of blood glucose was determined in approximately $30 \%$ of the study patients $(18,25,24$ and 22 patients in the placebo and the $2-$, 4- and 6-mg rosiglitazone twice-daily groups, respectively). The numbers of patients in each group are not exactly equal because some patients were missing baseline or end-point data. Significant reductions in postprandial glucose (PPG) AUCs were observed in all rosiglitazone treatment groups compared with both placebo and baseline (Fig. 2). Peak PPG concentrations were generally observed 90 min after a meal. Mean peak PPG concentrations at week 8 were $20 \mathrm{mmol} / \mathrm{l}$ in the placebo-treated group and $14 \mathrm{mmol} / 1$ for rosiglitazone-treated patients.

The change in PPG from week 0 to week 8 in the placebo and the rosiglitazone 4-mg twice-daily groups was examined by evaluating the AUC of PPG normalised to the time zero (fasting) value (Fig.3). The area under the PPG curve was calculated from the time of the meal to $180 \mathrm{~min}$ after the meal. In placebo-treated patients, the area under the PPG curve did not change between baseline and week 8 , whereas rosiglitazone 4-mg twice daily reduced area under the PPG curve by 19\% (95\% confidence interval $[\mathrm{CI}],-36.6 \%$ to $-2.0 \%$ ) from baseline to week 8 . No change in postprandial AUC insulin or AUC triglycerides occurred in rosiglitazone-treated patients between week 0 and week 8 .

Safety and tolerability. More rosiglitazone patients (87\%) than placebo patients $(76 \%)$ completed the study. Approximately $9.3 \%$ of patients in the placebo group and $5.1 \%, 4.2 \%$ and $3.8 \%$ of patients in the $2-$, 
Table 4. Changes in fasting lipid variables

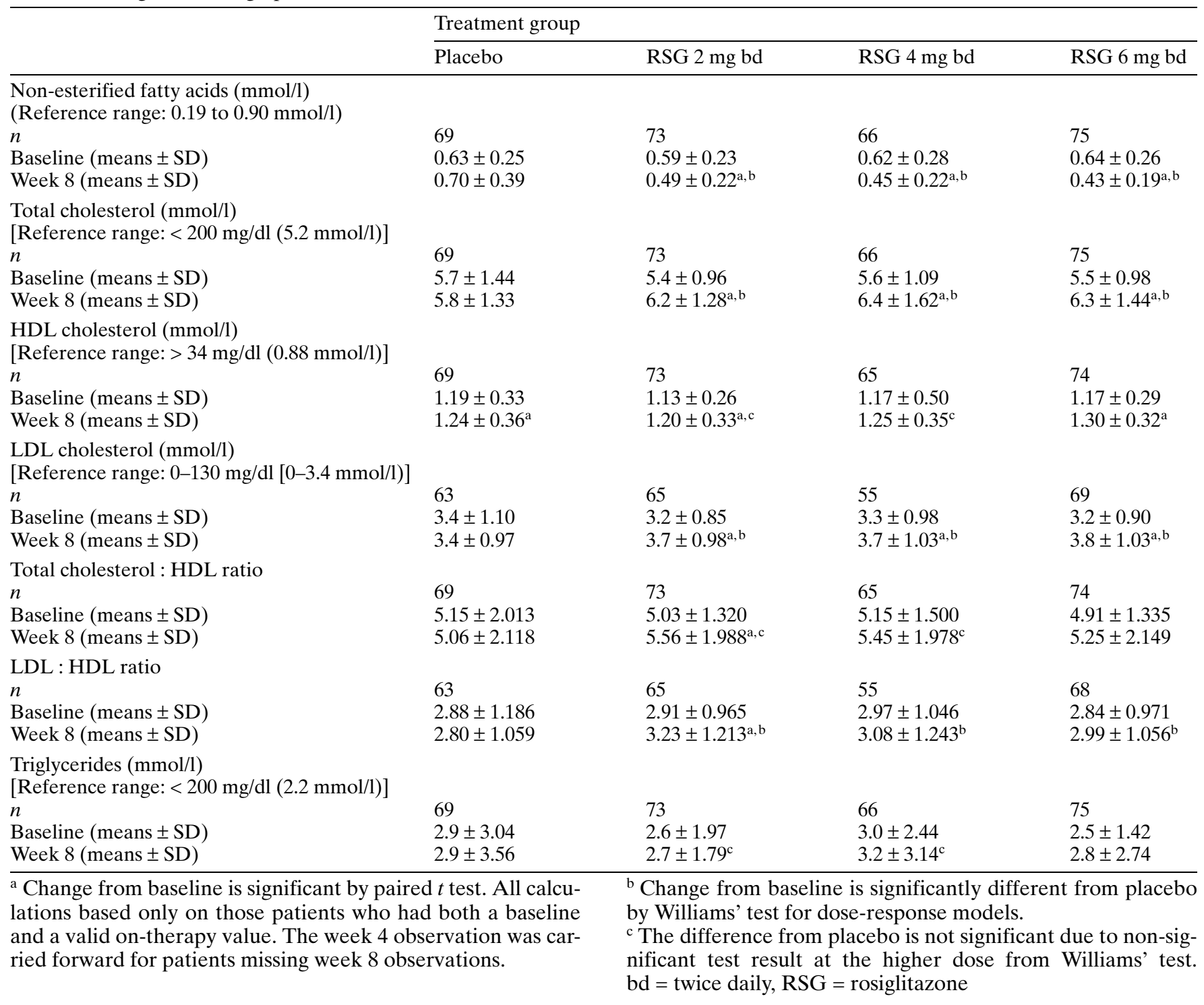

4- and 6-mg rosiglitazone twice-daily treatment groups, respectively, withdrew from the study because of an adverse event. Less than $2 \%$ of rosiglitazone patients withdrew for lack of efficacy compared with $5 \%$ of placebo patients.

The proportion of patients with at least one adverse event was similar in the four groups: $56.4 \%$, $54.9 \%$ and $49.4 \%$ in the rosiglitazone $2-$, $4-$ and 6-mg twice-daily treatment groups, respectively, compared with $49.3 \%$ in the placebo group. There were no notable changes in liver function tests in rosiglitazone-treated or placebo-treated patients. The week8 mean AST values for the placebo and rosiglitazone 2-, 4- and 6-mg groups were $18.7 \pm 8.36,19.1 \pm 8.23$, $17.5 \pm 9.96$ and $15.6 \pm 5.05 \mathrm{U} / 1$, respectively; the week-8 mean ALT values were $23.4 \pm 12.28$, $21.4 \pm 10.62,20.6 \pm 15.85$ and $16.2 \pm 6.04 \mathrm{U} / 1$, respectively. One patient in the $6-\mathrm{mg}$ twice-daily group withdrew because of oedema. Seven patients in the 6-mg twice-daily group, three in the 4-mg twice-daily group and one in the 2-mg twice-daily group had decreases in haemoglobin or haematocrit that were potentially clinically meaningful, but no patient withdrew from the study because of these adverse events or laboratory abnormalities. Mean changes from baseline in haemoglobin levels were $-0.2 \pm 0.37$, $-0.5 \pm 0.38$ and $-0.6 \pm 0.45 \mathrm{mmol} / \mathrm{l}$ for the $2-\mathrm{mg}$, $4-\mathrm{mg}$ and 6-mg rosiglitazone treatment groups, respectively, and mean changes from baseline in haematocrit levels were $-1.2 \% \pm 1.91 \%,-2.5 \% \pm 1.88 \%$ and $-3.0 \% \pm 2.29 \%$, respectively. Small increases in body weight were noted in the rosiglitazone 4- and 6-mg twice-daily groups $(0.7 \pm 2.34 \mathrm{~kg}$ and $1.5 \pm 2.45$ $\mathrm{kg}$, respectively). 

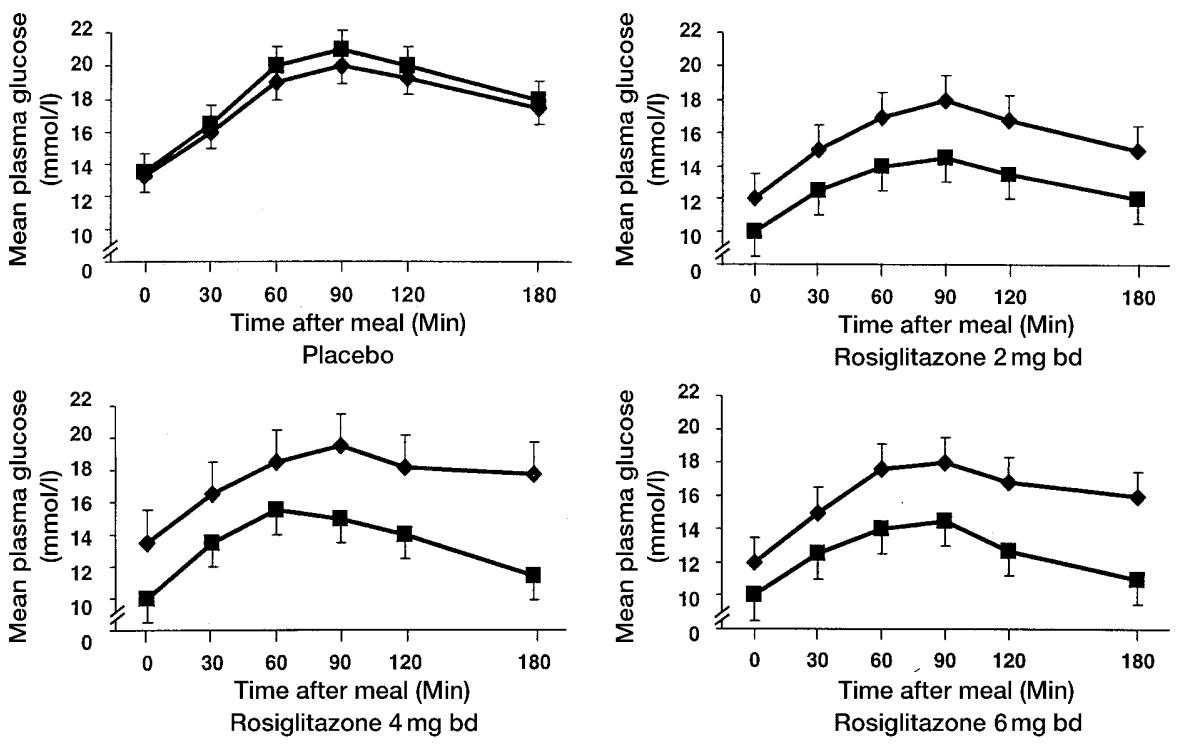

Fig. 2. Effect of rosiglitazone or placebo on plasma glucose concentrations before and after a standard meal. Error bars $=\mathrm{SE}, \diamond$ week 0 , $\square$ week 8, bd $=$ twice daily

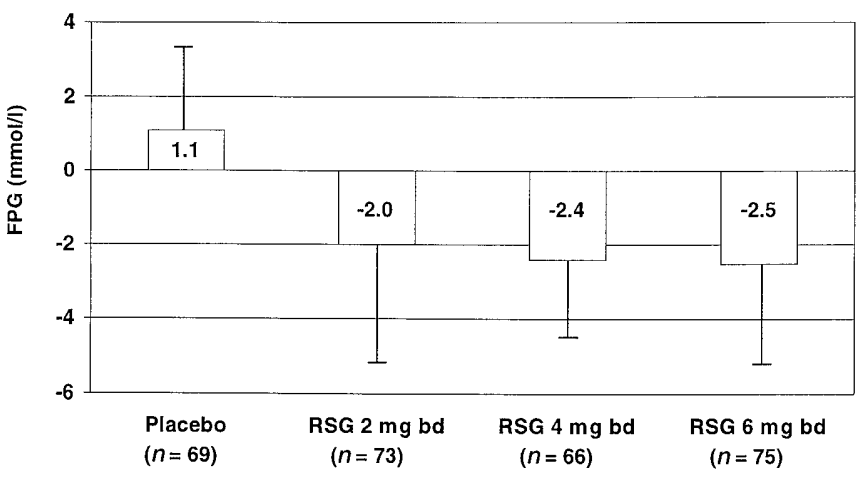

Fig. 3. Postprandial plasma glucose excursion normalised to baseline in the placebo and rosiglitazone 4-mg twice-daily groups. Error bars $=\mathrm{SE}, \diamond$ week 0 , week 8 , bd = twice daily

groups. Significant effects on $\mathrm{HbA}_{1 \mathrm{c}}$ are not expected in an 8-week study. Despite the relatively short duration of this study, some reduction in the $\mathrm{HbA}_{1 \mathrm{c}}$ values was, however, observed. The effects of the 4-mg twice-daily dose were similar to those of the $6-\mathrm{mg}$ twice-daily dose, suggesting that the top of the doseresponse curve had been attained. Treatment with rosiglitazone at all doses significantly reduced fasting plasma glucose concentrations and postprandial glucose peak concentrations and excursions, which appears to be primarily reflective of glucose disposition. The reduction in postprandial glucose with rosiglitazone is noteworthy because high postprandial glucose concentrations are associated with an increased risk of coronary artery disease [9-11], microvascular complications $[12,13]$ and a 1.5 -fold to threefold increase in the risk of sudden cardiac death [14].

Improved glycaemic control occurred in rosiglitazone-treated patients without an accompanying increase in fasting or postprandial insulin concentrations. This finding is consistent with the concept that rosiglitazone improves peripheral insulin sensitivity and subsequently improves glucose uptake by skeletal muscle [15]. It has been previously shown that $80-90 \%$ of insulin-stimulated glucose uptake occurs in skeletal muscle [15].

Rosiglitazone reduced NEFA concentrations.

This dose-ranging study clearly shows that rosiglitazone given as monotherapy in doses of 2,4 and $6 \mathrm{mg}$ twice daily reduces fasting and postprandial glucose concentrations. Reductions in fasting plasma glucose were observed in rosiglitazone-treated patients at the first office visit after the initiation of therapy, after only 4 weeks of treatment; additional improvement in glycaemic control was seen in rosiglitazonetreated patients after 8 weeks. Consistent with the short duration of treatment, reductions in fasting and postprandial glucose concentrations were best reflected in statistically significant differences in fructosamine between the placebo and rosiglitazone Raised NEFA concentrations are associated with the development of hypertension [16]. They are also known to increase insulin resistance in skeletal muscle and inhibit beta-cell function [17-20].

Total cholesterol and LDL cholesterol concentrations were increased by $6 \mathrm{mg}$ twice-daily rosiglitazone; the total cholesterol/HDL cholesterol ratio, considered a strong marker of cardiovascular risk, was, however, unchanged. These lipid changes have also been seen with other thiazolidinedione therapy [21]. Serum triglyceride concentrations, both fasting and postprandial, were unchanged by rosiglitazone treatment. 
Rosiglitazone was well tolerated. The overall frequency of adverse events was similar between the rosiglitazone and placebo treatment groups. No rosiglitazone patient withdrew from the study because of abnormal laboratory test values.

Rosiglitazone improved fasting and postprandial glucose concentrations compared with baseline and with placebo in patients with Type II diabetes. Doseresponse data reported here suggest that rosiglitazone will be useful at doses of $4 \mathrm{mg}$ to $8 \mathrm{mg}$ a day. Rosiglitazone reduced insulin and NEFA in addition to lowering glucose concentrations. The actions of rosiglitazone in this study are consistent with its activity as an insulin-sensitising agent and support its benefits in the treatment of Type II diabetes.

\section{Participating investigators and centres}

B Amin, Midtown Family Medicine, Columbia, SC; L Blonde, Alton Ochsner Medical Foundation, New Orleans, La; T Brodie, Vista Medical Research, Mesa, AZ; H Cathcart, Northside Internal Medicine, Spokane, Wash; PK Champion, KelseySeybold Clinic - Medical Center, Houston, Tex; E Conrad, Lake Medical Clinic, Lake Oswego, Or; M Farmer, Clinical Studies, Florida, St. Petersburg, Fla; J Gabriel, North Hills Medical Research, North Richland Hills, Tex; G Gewirtz, Joslin Center for Diabetes at Saint Barnabas, West Orange, NJ; WJ Henry, MeDQuest, Greer, SC; JR Herbst, Hill Top Research, Portland, Or; KS Hershon, North Shore Diabetes \& Endocrine Associates, New Hyde Park, NY; S Hsi, Albuquerque, NM; J Isaacsohn, Metabolic and Atherosclerosis Research Center, Cincinnati, Oh; D Iverson, Cascade Physicians, Portland, Or; C Kilo, Kilo Clinical Research, St. Louis, Mo; A Koff, GHSD Parkview Hospital, Philadelphia, Pa; A Lewin, National Research Institute, Los Angeles, Calif; A Licata, The Cleveland Clinic Foundation, Cleveland, Oh; J Mersey, Mersey Clinical Research, Baltimore, Md; W Mullican, MediSphere Medical Research Center, Evansville, Ind; L Mulmed, Abbott Northwestern Diabetes Center, Minneapolis, Minn; JV Murray, Hill Top Research, St. Petersburg, Fla; PA Orlander, University of Texas Health Science Center, Houston, Tex; RG Paolino, Health Center, Bensalem, Pa; M Patel, Taunton Health Clinic, Taunton, Ma; P Raskin, University of Texas Southwestern Medical Center at Dallas, Dallas, Tex; G Redmond, Center for Health Studies, Cleveland, Oh; J Reusch, VA Medical Center, Section of Endocrinology, 111H University Hospital, Denver, Colo; S Rosenblatt, The Irvine Clinical Research Center, Irvine, Calif; C Stuart, University of Texas Medical Branch at Galveston, Galveston, Tex; D Sugimoto, Cedar-Crosse Research Center, Chicago, Ill; R Weinstein, Diablo Clinical Research, Walnut Creek, Calif; T Williams, Clinical Studies, Arizona, Peoria, Ariz; J Wilson, Advanced Research Management, LP (ARM), Seattle, Wash; K Wingert, Community Medical Providers, Clovis, Calif

\section{References}

1. DeFronzo RA (1988) The triumvirate: $\beta$-cell, muscle, liver. A collusion responsible for NIDDM. Diabetes 37: 667-687

2. DeFronzo RA (1992) Pathogenesis of Type 2 (non-insulin dependent) diabetes mellitus: a balanced overview. Diabetologia 35: 389-397
3. Saltiel AR, Horikoshi H (1995) Thiazolidinediones are novel insulin-sensitizing agents. Curr Opin Endocrinol Diabetes 2: 341-347

4. Lehmann JM, Moore LB, Smith-Oliver TA, Wilkison WO, Willson TM, Kliewer SA (1995) An antidiabetic thiazolidinedione is a high affinity ligand for peroxisome proliferator-activated receptor $\gamma(\operatorname{PPAR} \gamma)$. J Biol Chem 270: 12953-12956

5. Willson TM, Cobb JE, Cowan DJ et al. (1996) The structure-activity relationship between peroxisome proliferator-activated receptor $\gamma$ agonism and the antihyperglycemic activity of thiazolidinediones. J Med Chem 39: 665-668

6. Patel J, Anderson RJ, Rappaport EB (1999) Rosiglitazone monotherapy improves glycaemic control in patients with type 2 diabetes: a twelve-week, randomized, placebo-controlled study. Diabetes, Obesity and Metabolism 1: 165-172

7. National Diabetes Data Group (1979) Classification and diagnosis of diabetes mellitus and other categories of glucose intolerance. Diabetes 28: 1039-1057

8. Friedewald WT, Levy RI, Fredrickson DS (1972) Estimation of the low density lipoprotein cholesterol in plasma without use of the preparative ultracentrifuge. Clin Chem 18: 499-502

9. Mooradian AD, Thurman JE (1999) Drug therapy of postprandial hyperglycaemia. Drugs 57: 19-29

10. Hanefeld M, Temelkova-Kurktschiev T (1997) The postprandial state and the risk of atherosclerosis. Diabet Med 14 [Suppl 3]:S6-S11

11. Jackson CA, Yudkin JS, Forrest RD (1992) A comparison of the relationships of the glucose tolerance test and the glycated hemoglobin assay with diabetic vascular disease in the community. The Islington Diabetes Survey. Diabetes Res Clin Pract 17: 111-123

12. Ohkubo Y, Kishikawa H, Araki E et al. (1995) Intensive insulin therapy prevents the progression of diabetic microvascular complications in Japanese patients with noninsulin-dependent diabetes mellitus: a randomized prospective 6-year study. Diabetes Res Clin Pract 28: 103-117

13. Haller H (1997) Postprandial glucose and vascular disease. Diabet Med 14 [Suppl 3]:S50-S56

14. Curb JD, Rodriguez BL, Burchfiel CM, Abbott RD, Chiu D, Yano K (1995) Sudden death, impaired glucose tolerance, and diabetes in Japanese American men. Circulation 91: 2591-2595

15. Saltiel AR, Olefsky JM (1996) Thiazolidinediones in the treatment of insulin resistance and type II diabetes. Diabetes 45: 1661-1669

16. Fagot-Campagna A, Balkau B, Simon D et al. (1998) High free fatty acid concentration: an independent risk factor for hypertension in the Paris Prospective Study. Int J Epidemiol 27: 808-813

17. Reaven GM (1988) Banting Lecture 1988. Role of insulin resistance in human disease. Diabetes 37: 1595-1607

18. Dresner A, Laurent D, Marcucci M et al. (1999) Effects of free fatty acids on glucose transport and IRS-1-associated phosphatidylinositol 3-kinase activity. J Clin Invest 103: 253-259

19. McGarry JD, Dobbins RL (1999) Fatty acids, lipotoxicity and insulin secretion. Diabetologia 42: 128-138

20. Paolisso G, Howard BV (1998) Role of non-esterified fatty acids in the pathogenesis of type 2 diabetes mellitus. Diabet Med 15: 360-366

21. Kumar S, Boulton AJM, Beck-Nielsen H et al. (1996) Troglitazone, an insulin action enhancer, improves metabolic control in NIDDM patients. Diabetologia 39: 701-709 